\title{
Selective serotonin reuptake inhibitors were less cost effective than initial treatment with tricyclic antidepressants
}

Canadian Coordinating Office for Health Technology Assessment. Selective serotonin reuptake inhibitors (SSRIs) for major depression. Part II. The cost-effectiveness of SSRIs in treatment of depression. Ottawa: Canadian Coordinating Office for Health Technology Assessment, 1997.

\section{Objective}

To compare the cost effectiveness of selective serotonin reuptake inhibitors (SSRIs) and tricyclic antidepressants (TCA) as first and second line treatment for depression.

\section{Design}

Cost utility analysis from a government payer perspective using a decision model to compare 3 treatment paths over 9 months. The model included data from meta-analyses and a randomised controlled trial. Patient preferences were incorporated using the data from 1 study of 70 patients with major depressive disorder.

\section{Setting}

Canada.

\section{Patients}

3 theoretical cohorts of patients with major depression.

\section{Intervention}

The 3 strategies were initial SSRI treatment with dropouts receiving TCAs after 3 months; initial TCA treatment with dropouts receiving SSRIs after 3 months; and treatment with TCAs only.

\section{Main costs and outcome measures}

The incremental probability of success, cost of treating a patient, utility, and cost per quality of life adjusted year (QALY) gained over 3 and 9 months. Only direct costs (Canadian dollars) were included in the analysis.

\section{Main results}

Initial SSRI treatment for 3 months had a $5.6 \%$ higher probability of success than initial treatment with both TCA and TCA alone but increased costs by $\$ 44$. Initial SSRI treatment over 9 months had a $0.3 \%$ higher probability of success than initial TCA treatment, but increased costs by $\$ 110$. Compared with TCA treatment alone, initial SSRI treatment over 9 months had a 9\% higher success probability and decreased costs by $\$ 406$. The incremental utility of initial SSRI treatment over 9 months was 0.039 compared with initial TCA treatment and $0.042 \mathrm{com}-$ pared with TCA alone. The cost per QALY gained over 9 months was $\$ 2818$ for initial SSRI treatment compared with initial TCA treatment. Sensitivity analyses varied the dropout rates, relapse rates, physician costs, psychiatric costs, drug costs, length of stay in hospital, and utility values. When the lowest dropout rate for TCA was used, initial TCA treatment had a $0.5 \%$ greater success rate than initial SSRI treatment over 3 months. When desipramine and sertraline costs were used, initial SSRI treatment over 3 months was less expensive than initial TCA treatment and reduced the cost of each QALY gained. The costs for initial SSRI treatment decreased over time when the highest dropout rate for TCA was used, the length of hospital stay was high, sertraline and desipramine costs were used, or the dropout rates from 1 meta-analysis were used (TCA 54\%, SSRI 42\%).

\section{Conclusion}

Selective serotonin reuptake inhibitors (SSRIs) as first line treatment for depression were more cost effective than tricyclic antidepressants (TCAs) alone, but less cost effective than TCAs as first line treatment with SSRIs for dropouts.

Sources of funding: Federal, Provincial, and Territorial Governments of Canada.

For article reprint:J F Baladi, 110-955 Green Valley Crescent, Ottawa, Ontario K2C 3V4, Canada. Fax +16132265392

Abstract and commentary also published in Evidence-Based Medicine 1998 May-Jun.

(commentary continued from page 50)

Whereas previous meta-analyses generally agreed with the conclusions of the CCOHTA's first report, their economic modelling will be more contentious. To explore the cost of prescribing SSRIs $v$ tricyclics, the CCOHTA examined 3 treatment protocols: (1) give tricyclics only; (2) give tricyclics first, then give an SSRI if the patient cannot tolerate it; and (3) start with an SSRI and then go onto a tricyclic. Most previous economic analyses based on decision modelling have favoured SSRIs. This report suggests that using tricyclics alone is not cost effective, but starting tricyclics and moving to SSRIs if not tolerated (second option) is more cost effective than the third option.

There is much here to commend. The report used data derived from randomised trials wherever possible. The authors presented a more realistic therapeutic alternative to that used in many other economic models in this field. The main problem with the approach is that it is often based on multiple assumptions. ${ }^{2}$ The authors partially overcame this by presenting a number of sensitivity analyses with different dropout rates and tricyclic doses, and the main findings are reasonably robust. However, they still had to cobble together results of other studies to estimate resource use on either treatment. They also assumed that rates and costs of overdose, suicide, and road traffic accidents are similar on the 2 treatments-outcomes which are always difficult to model, but are probably rarer on SSRIs. Finally, costs are not constant between countries: what applies in one healthcare setting may not in another.
Whereas efficacy data are likely to be generalisable, cost effectiveness data are not.

Despite these caveats, these are important and useful reports. SSRIs have undoubted advantages, and as their price falls they may become first line treatment. For the time being, however, tricyclics remain the most cost effective first line treatment for depression.

Matthew Hotopf, MBBS, MRCPsych, MSc Institute of Psychiatry London, UK

1 Paykel ES, Priest RG. Recognition and management of depression in general practice: consensus statement. BMJ 1992;305:1198-1202.

2 Hotopf M, Lewis G, Normand C. Are SSRIs a cost-effective alternative to tricyclics? Br J Psychiatry 1996;168:404-9. 